\title{
11. Theatre and Health: Origins, Areas and Perspectives
}

\author{
Alessandra Rossi Ghiglione \\ doi.org/10.3280/oa-637-11
}

\section{The Current Challenge}

COVID-19 has clearly demonstrated how a life of relationships and active cultural participation is essential to the psychosocial and physical well-being of people and communities. Given that they are eminently social and symbolic animals, human beings need to express themselves and create and experience meanings through actions involving other human beings and interacting with the environment in which they live. The pandemic has had a greater negative impact on the health of the disadvantaged and vulnerable (Bambra et al., 2020; "COVID-19 casts light", 2020), for whom the lack of opportunities and of access to social and cultural resources is greater (Pitas and Ehmer, 2020; Van Bavel et al., 2020). Additionally, COVID-19 has highlighted the fundamental role of religious and civil rituality in the elaboration of mourning and in the production of collective meanings able to generate shared values and visions of possible futures following the experience of death.

\section{Origins and Evolution of the Relationship Between Art and Health}

In the ancient world the healing scene was integrated: medicine, spirituality and rite constituted a unitary knowledge geared towards the salus, the healing of mankind as a whole. According to the Hippocratic school $\left(5^{\text {th }}-4^{\text {th }}\right.$ century B.C.), health did not concern only the biological dimension of the individuals, but it included their lifestyle and their natural and social environment. Hippocrates was the first to point to the theatre as a practice of health, inviting his patients to treat themselves with a vision of tragedy and comedy (Cosmacini, 1997).

In the following centuries, art and care were separated. The notion of health was reduced to the mere absence of illness. Medicine became a cure for the physical body alone. Although now outdated, this so-called biomedical approach still informs many health systems throughout the world, as well as the basic training of professionals and part of scientific research.

The questioning of the Cartesian dualism of body and mind, which began at the end of the $19^{\text {th }}$ century, and the birth of the social sciences - anthropology, 
sociology, psychology, new pedagogy - relaunched the role of culture in shaping human experience and paved the way for a paradigm shift in medicine. In 1977, the US psychiatrist George L. Engel opposed to the biomedical model a new biopsychosocial model, highlighting the decisive role played by psychic and social factors in health (Engel, 1977). To this model refer today the new branches of medicine, such as PNEI or Psychoneuroendocrineimmunology (Bottaccioli, 2014), epigenetics ${ }^{52}$ and neuroaesthetics, which, following the discovery of the role of mirror neurons (Rizzolatti and Sinigaglia, 2006), concentrates on what happens to people in the experiences of artistic fruition and creation (Gallese, 2010 and 2013).

In parallel with developments in scientific research, from the post-war period, the World Health Organization has been gradually endorsing the change in the concept of health and expressing the new principles on which international health policies are based.

The following principles are basic to the happiness, harmonious relations and security of all peoples: Health is a state of complete physical, mental and social well-being and not merely the absence of disease or infirmity. The enjoyment of the highest attainable standard of health is one of the fundamental rights of every human being without distinction of race, religion, political belief, economic or social condition. The health of all peoples is fundamental to the attainment of peace and security and is dependent upon the fullest co-operation of individuals and States ${ }^{53}$.

The 19 December 1946 Declaration - included in the preamble of the WHO Charter - formulates the current definition of health and, paying attention to the right to health and the interconnection between health, peace and security, lays the basis for subsequent declarations on the issues of health promotion (Lemma, 2005), intersectoral approaches to health policies ${ }^{54}$ and equity (Arcaya M.C., Arcaya A.L. and Subramanian, 2015).

In 1986 with the publication of the WHO's Ottawa Charter for Health Promotion (WHO, 1986), the idea of health as a dynamic state determined by a plurality of factors was ratified with greater clarity. Along with factors that cannot be modified - biological and genetic - there are numerous other health factors that are modifiable and upon which it is possible to act both preventatively, with health promotion, and therapeutically. The focus is on

\footnotetext{
${ }^{52}$ Epigenetics is gathering evidence on the possibility that human behaviour, including that stimulated by cultural and artistic practices, can modify genetic factors (Martienssen, Riggs and Russo, 1996).

53 The constitution was ratified on 7 April 1948 (WHO, 2002). The same definition is also proposed in the Health Promotion Glossary (WHO, 1998).

54 This approach was ratified in 2013 in the WHO declaration of Helsinki (WHO, 2014).
} 
"health resources" from which we can take action on two levels: on an individual one, by strengthening knowledge and «health skills» (empowerment) (Laverack, 2005) and developing processes that «enable people to gain greater control over the determinants of their health and improve it» (WHO, 1986); and on a socio-environmental level, favouring the creation of «healthcare opportunities» based on an approach from below, generated by the community itself, which is integrated into the local traditions of its members (IUHPE, 2008; WHO, 2020).

The paradigm shift is fundamental: from the centrality of disease treatment to the strategic role of health education and health promotion, from the biomedical approach to a «salutogenic» approach (Antonovsky, 1996) that acts effectively on the «determinants of health». It is in this perspective that artistic actions are recognised for their ability to impact on the social (Cooper et al., 1999; Allen J. and Allen M., 2016) and cultural (Abel, 2007 and 2008) determinants of health.

Social capital (Putman, 2000), that is, all the social resources available to a person or a community, has a positive correlation with both the well-being of a person or a community and their life expectancy (Hyyppä and Mäki, 2003). Evidence has also been gathered on the correlation between cultural capital (Throsby, 1999), in particular participation in cultural activities, and subjective well-being (Grossi et al., 2011) and life expectancy (Bygren, Konlaan and Johansson, 1996; Johansson, Konlaan and Bygren, 2001).

Research on the impact of culture and art on health reached a milestone on 19 November 2019 with the publication by WHO Europe of a review of world literature over the last twenty years (Fancourt and Finn, 2019). The research affirms the contribution of the arts to mental and physical health in the four areas of prevention, promotion, management and treatment and calls for health policies to take this into account. The benefits of the arts under consideration (performing arts, visual arts, literature, culture and digital arts) are found both in active participation and in passive and spectatorial forms. The effectiveness of artistic practices can be traced back to three main characteristics: the holistic perspective, the multimodal dimension, the ability to link the individual health dimension with the social context ${ }^{55}$.

\footnotetext{
${ }^{55}$ According to this research, the answers produced are psychological, physiological, social and behavioural and depend on nine types of activation typical of artistic practices, which the research indicates: aesthetic engagement, involvement of the imagination, sensory activation, evocation of emotion, cognitive stimulation, social interaction, physical activity, engagement with themes of health, interaction with health-care settings.
} 


\section{The Power of Theatre in Building Personal and Community Health}

Both in the field of promotion (Matricoti, 2010; Rossi Ghiglione and Tortone, 2020) and of treatment (Brodzinski, 2010), theatre is nowadays recognised as an effective practice important for health challenges: healthy ageing and mental health of the elderly, health education and development, vulnerability, social inclusion, care and training of health personnel (Pontremoli and Rossi Ghiglione, forthcoming). Many are the specific impacts that the theatre produces and that contribute to the construction of individual and social health in these areas. They concern: the strengthening of life skills (WHO, 1994) (with particular regard to empathy, effective communication, emotion management and collaboration) and of mnestic and cognitive skills; the development of social value and social capital; the organic physical reactivation and the integration of body and mind; the inclusion and acceptance of diversity; the ethical reflection and the strengthening of critical learning; the promotion of resilience and stress management.

The original anthropological matrix of the theatre combines the ritual (Turner, 1982), ludic and symbolic dimensions. Being a complex multimodal and multilinguistic cultural device, which favours active participation (Rossi Ghiglione, 2014), theatre - intended both as what is acted and what is watched - activates men on many levels and creates many dynamic connections (involving sensations, emotions, cognitions, relationships) (Rossi Ghiglione, 2019a). This device originated in Greek civilisation, where theatre was as much a social and civil care process as a pedagogical and andragogical arena (Knowles, 1980).

Contemporary theatrical practices oriented towards well-being and health have their roots in the $20^{\text {th }}$ century theatre revolution. Copeau and the master pedagogues of the beginning of the last century (Cruciani, 1971 and 1985) were the first to refocus their attention on the ethical dimension of theatre, its role in the community, its ability to build groups and to connect different cultures (De Marinis, 2011). They also developed a rich repertoire of psychophysical and relational practices which is the basis of the techniques currently used in theatre and health. In the 1950s, and with greater effect in the 1960s and 1970s, a theatre movement emerged consisting of artists, intellectuals and pedagogues who chose to move out of the theatres - both the physical spaces and the commercial production models - and engage with citizens in processes of social and political exchange and change (De Marinis, 1983): Animazione Teatrale ${ }^{56}$ in France and Italy, community-based ${ }^{56}$ For an explanation of the meaning of Animazione teatrale see the Notes on Translations on
p. 7. The expression is used in this sense throughout the paper. 
theatre in Anglo-Saxon countries and South America $^{57}$, moved into communities and met disadvantaged groups with a social and political, rather than therapeutic objective.

Social and Community Theatre or Teatro sociale di comunita ${ }^{58}$ (Bernardi, 2004; Pontremoli, 2015; Rossi Ghiglione, 2019b) today, which in Italy reaped the legacy of the $20^{\text {th }}$ century theatre in its function of social care and cure, is well recognised even among the community based arts for the support of the psychosocial well-being of individuals and communities in emergency situations (Rossi Ghiglione and Schininà, 2019). The specific methodological and evaluative research on theatre and health which was launched in Turin in the early 2000s (Nicotera et al., 2009; Rossi Ghiglione, 2011a; Pagliarino, 2017) in partnership with health and university institutions, today constitutes an intersectorial heritage able to meet the challenges indicated by the 2018 European Agenda for Culture on social inclusion and community well-being and the more recent ones on combating the inequalities of the pandemic.

${ }^{57}$ Of interest are the experiences inspired by the pedagogist Freire and Boal's Theatre of the Oppressed, whose approach is widely used in health education.

58 For an explanation of the meaning of Teatro sociale di comunità see the Notes on Translations on p. 7. The expression is used in this sense throughout the paper. 